\title{
CATS, RABBITS, BIRDS, AND VIRUSES, OH MY! MODELLING THE CONSERVATION IMPLICATIONS OF A COMPLEX VIRUS RELEASE IN A PREDATOR-PREY SYSTEM
}

\author{
JEAN-PAUL R. SOUCY* \\ FACULTY ADVISOR: ROBERT SMITH?†
}

\begin{abstract}
Mathematical models have proven useful in planning conservation efforts for threatened species. In this paper, we develop a model based on the Macquarie Island ecosystem, where native seabirds were threatened by invasive pest species. In particular, European rabbits (Oryctolagus cuniculus) destroyed seabird nesting sites, while feral cats (Felis catus) preferentially hunted rabbits but also consumed seabirds. Management strategies included releasing a rabbit-killing disease and shooting feral cats. We investigate the interactions between species in the ecosystem as well as conservation practices using analytical techniques such as the basic reproductive ratio $\left(R_{0}\right)$ and partial rank correlation coefficient analysis. The results of this study reveal that the important factors to disease establishment change depending on the ecological interactions present in the system. Additionally, we show that the interaction between disease and predation sometimes produces surprising outcomes. Overall, the results of the study demonstrate the need for mathematical modelling in the conservation process in order to anticipate the complex responses of an ecosystem to management practices. We conclude with a brief list of considerations for conservation planners dealing with ecologically complex systems in the future.
\end{abstract}

Key words. Disease model, predator-prey, conservation

AMS subject classifications. 92D $30,92 \mathrm{D} 40,34 \mathrm{C} 60$

1. Introduction. Macquarie Island is a small strip of land nestled between New Zealand and Antarctica. At just under 50 square miles, this UNESCO World Heritage Site has been subject to numerous invasive species since it was first discovered two centuries ago [19]. These species include rodents, cats, rabbits, pigs, goats, weka, and other non-native animals. The inevitable victims of each new introduced pest are the island's native seabird populations. Although the island's pest eradication program has seen success in recent years [6], the island's history has been fraught with missteps that have only worsened the ecological situation $[1,6,19]$.

In 1978, the flea-borne Myxoma virus was released on Macquarie Island to control the population of European rabbits (Oryctolagus cuniculus). Rabbits threatened the nesting locations of native seabirds by decimating the vegetation of the island. Also present were feral cats (Felis catus) that preyed preferentially on rabbits, although they would also prey on seabirds in the absence of sufficient leporid prey. Thus the fate of the seabirds hung in a delicate balance: with too few rabbits, the cats preyed on the seabirds, but with too few cats, the rabbits would destroy the habitat the seabirds use to nest [1].

\footnotetext{
*Department of Biology and Department of Mathematics and Statistics, University of Ottawa, Ottawa, K1N 6N5, Canada (jsouc073@uottawa.ca).

$\dagger$ Department of Mathematics and Statistics and Faculty of Medicine, University of Ottawa, Ottawa, K1N 6N5, Canada (rsmith43@uottawa.ca).
} 
This was not the first time Myxoma virus had been used to control rabbit populations. In European rabbits, the virus causes the disease myxomatosis. Infected rabbits develop skin tumours and usually die within 8-12 days [12]. This virus was intentionally released in Australia and France in the 1950s and led to dramatic reductions of rabbit populations in these countries and other European nations. However, hostpathogen co-evolution and rising genetic resistance to the disease limits the disease's use as an ongoing control measure in Australia and other places [12].

The Macquarie Island ecosystem presents an interesting opportunity to model the effects of using a virus for the purpose of conservation in a complex predator-prey system. Myxoma virus is able to be spread either through direct contact with an infected rabbit or through contact with an infected flea vector [12]. Annual release of the virus was done from 1978 until stocks were depleted in the mid-2000s [17]. Brothers et al. (1982) (as cited in [8]) suggest that the reason for annual release was due to unfavourable environmental conditions. These factors and the aforementioned emergence of attenuated strains and genetic immunity make this a complicated disease system.

Mathematical models have previously been used to inform conservation decisions, for example during the successful restoration of the Channel Island fox [23]. This model aims to combine traditional predator-prey modelling (with rabbits and seabirds as prey and feral cats as predators) and disease modelling with declining disease mortality and/or host susceptibility (Myxoma virus in rabbits) in order to provide insight on management practices, including the use of diseases, in complex ecosystems like Macquarie Island.

In this paper, we analyse the model component-by-component in order to make inferences about the entire system, culminating with simulations of the full model. In section 2, we develop the Macquarie Island model by building on simple ecological and disease models. In section 3, we establish the minimum conditions allowing the potential long-term persistence of predators and disease in the absence of seabirds. In section 4, we demonstrate that ecological interactions modify the importance of the different parameters that determine whether a disease establishes or dies out. Section 5 shows the effects of rabbits on seabirds, providing context to the subsequent section. Section 6 explores the importance of considering all aspects of an ecosystem before making conservation decisions. Section 7 summarizes the conclusions of the model and provides practical suggestions for conservation planning.

We use several common disease-modelling techniques in this paper, including the Jacobian, $R_{0}$, and Latin hypercube sampling (LHS)/partial rank correlation coefficient (PRCC) analysis. Briefly, the Jacobian allows the conditions for the stability of the steady states of an ODE to be computed. $R_{0}$, the basic reproductive ratio, is a fundamental concept in epidemiology that represents the average number of secondary cases produced by a single infectious individual over their infectious lifetime in a completely susceptible population. $R_{0}$ acts as a threshold: if $R_{0}>1$, the disease is able to invade a susceptible population, if $R_{0}<1$, the disease cannot invade and dies out. There are many ways of calculating $R_{0}$ (with differing results), but all methods share this threshold at 1 [13]. In this paper, we use the next-generation method [7]. PRCC allows us to test the sensitivity of $R_{0}$ to changes in various model parameters, with parameter combinations selected from a defined range using LHS. The purpose of LHS is to explore the whole parameter space with the least number of simulations. The final output is a diagram showing the positive or negative effects (and their relative magnitudes) on $R_{0}$ of increasing each parameter. The aim of this paper is to apply these tools to the Macquarie Island model to provide insight on conservation 
strategies in systems with complex ecological interactions.

2. Model Development. We begin with a step-by-step development of the model.

The model builds upon the basic Lotka-Volterra predator-prey equations [14] and the ubiquitous SIR model of disease:

$$
\begin{aligned}
& \frac{d x}{d t}=\alpha x-\beta x y \\
& \frac{d y}{d t}=\delta x y-\gamma y
\end{aligned}
$$

$$
\begin{aligned}
\frac{d S}{d t} & =-\beta I S \\
\frac{d I}{d t} & =\beta I S-\gamma I \\
\frac{d R}{d t} & =\gamma I
\end{aligned}
$$

In the predator-prey model, prey $(x)$ grow exponentially according to their growth rate $(\alpha)$ and are consumed by predators $(y)$, where $\beta$ is the predation rate, $\delta$ is the growth rate from predation, and $\gamma$ is the natural mortality rate of the predator. In general, this model predicts sinusoidal cycles for both predator and prey, where the predator's cycle lags behind the prey's. In the SIR model, susceptible individuals $(S)$ are infected through contact with infected individuals $(I)$, which is influenced by the contact rate $(\beta$, a product of encounter rate and probability of infection upon encounter). Individuals recover (or are otherwise "removed") $(R)$ after $\gamma^{-1}$ time units.

In the present model, susceptible and infected rabbits ( $S$ and $I$, respectively) are prey and cats $(C)$ are predators (see Table 1 for a full list of model variables and parameters). The first modification to the basic model is to make rabbit growth logistic; that is, the population of rabbits proceeds asymptotically toward a carrying capacity $K$, with maximum per capita growth rate $\alpha$. This reflects both the European rabbit's capacity for explosive growth and the very real resource and space limitations of the modestly-sized Macquarie Island $\left(128 \mathrm{~km}^{2}\right)$. We assume rabbits are born sexually mature, as rabbit kittens reach adulthood after only 3-6 months. A key feature of the logistic growth equation is that per capita growth rate declines linearly as the number of individuals increases, whereas population rate of change is parabolic, peaking when the population size is $K / 2$. In essence, logistic growth assumes perfectly density-dependant reproduction. First-year mortality of rabbits in the wild can be as high as $90 \%$ [26]. Since only sexually mature rabbits are represented in the model, density-dependant growth effectively accounts for most natural mortality and mortality before maturity, allowing us to neglect a natural death term for uninfected rabbits. This gives us:

$$
\frac{d S}{d t}=\alpha S\left(1-\frac{S}{K}\right)
$$

Next, we add disease dynamics, beginning with $\beta$, the contact rate of infected rabbits. The mean survival time (and thus infectious period) of infected rabbits is $\mu_{I}{ }^{-1}$. The literature fails to mention transplacental (vertical) transmission of Myxoma virus in rabbits, so we assume all rabbits are born uninfected. We add a function $f(t)$ to represent the decay in rabbit susceptibility to infection (due to genetic immunity and the emergence of benign strains). Fleas are not modelled explicitly, instead we model Myxoma transmission from fleas to rabbits with the function $g(t)$. Finally, 


\begin{tabular}{|c|c|c|}
\hline Symbol & Definition & Unit \\
\hline$B$ & Number of seabirds & birds \\
\hline$S$ & Number of susceptible rabbits & rabbits \\
\hline$I$ & Number of infected rabbits & rabbits \\
\hline$C$ & Number of cats & cats \\
\hline$\Lambda$ & Constant birth rate of seabirds & birds years ${ }^{-1}$ \\
\hline$\alpha$ & Maximum per capita growth rate per susceptible rabbit & years $^{-1}$ \\
\hline$K$ & Carrying capacity of rabbit population & rabbits \\
\hline$\beta$ & Contact rate of an infected rabbit & rabbits $^{-1}$ years $^{-1}$ \\
\hline$\sigma$ & Maximum effectiveness of flea transmission & years $^{-1}$ \\
\hline$\delta$ & Minimum effectiveness of flea transmission (proportion of max.) & dimensionless \\
\hline $\bar{\Omega}$ & Maximum decrease in seabird births by rabbits (by division) & dimensionless \\
\hline$\mu_{B}$ & Mortality rate of seabirds & years $^{-1}$ \\
\hline$\mu_{I}$ & Mortality rate of infected rabbits & years $^{-1}$ \\
\hline$\mu_{C}$ & Mortality rate of cats & years $^{-1}$ \\
\hline$p_{B}$ & Predation rate on seabirds & rabbits cats ${ }^{-1}$ years $^{-1}$ \\
\hline$p_{S}$ & Predation rate on susceptible rabbits & cats $^{-1}$ years $^{-1}$ \\
\hline$p_{I}$ & Predation rate on infected rabbits & cats $^{-1}$ years $^{-1}$ \\
\hline$e_{B}$ & Seabird conversion efficiency (cats born per seabird consumed) & cats birds ${ }^{-1}$ \\
\hline$e_{R}$ & Rabbit conversion efficiency (cats born per rabbit consumed) & cats rabbits $^{-1}$ \\
\hline$r$ & Long-term proportion of rabbits susceptible to disease & dimensionless \\
\hline$x$ & Time for rabbit susceptibility to disease to decay by half & years \\
\hline$k_{B}$ & Constant for logarithmic effect of rabbits on seabird births & rabbits \\
\hline
\end{tabular}

TABLE 1

List of model variables and parameters with definitions and units.

we introduce predation by cats, with separate predation rates on infected $\left(p_{I}\right)$ and uninfected $\left(p_{S}\right)$ rabbits. Although diseased rabbits should be easier to catch than healthy rabbits, they may also be less likely to leave their burrows, so the relative values for $p_{I}$ and $p_{S}$ are not immediately obvious.

$$
\begin{aligned}
& \frac{d S}{d t}=\alpha S\left(1-\frac{S}{K}\right)-p_{S} S C-f(t) \beta S I-f(t) g(t) \sigma S \\
& \frac{d I}{d t}=f(t) \beta S I+f(t) g(t) \sigma S-p_{I} I C-\mu_{I} I
\end{aligned}
$$

Equation (2.5) gives $f(t)$, the disease susceptibility function, which declines asymptotically through time from 1 (completely susceptible) at time $t \psi=0$ toward some minimum susceptible proportion $r$, where $0<r<1$. This function also has the property that it decays by half by time $x$. Equation (2.6) gives $g(t)$, the flea transmission function, which varies seasonally in a sinusoidal manner. This function has a period of 1 year, representing the annual re-release of the virus. Transmission effectiveness oscillates between $\sigma \psi($ maximum effectiveness) and $\delta \sigma$, where $\delta \psi$ is some proportion of the maximum effectiveness. Maximum transmission effectiveness is achieved halfway through the period. Of course, the proportion of rabbits susceptible to infection by fleas is modulated by $f(t)$. 


$$
\begin{gathered}
f(t)=\frac{(1-r) / r}{(1-r) / r+e^{q t}}+r \\
q=\frac{1}{x} \ln \left(\frac{1+r}{r}\right) \\
g(t)=-\left(\frac{1-\delta}{2}\right) \cos (2 \pi t)+\frac{1+\delta}{2}
\end{gathered}
$$

Next, we consider the seabirds $(B)$ of Macquarie Island, which we abstract as one homogeneous population. For simplicity, we assume the seabird birth rate $(\Lambda)$ is independent of population size. The seabird population is modulated by three things: habitat destruction by rabbits, predation by cats, and natural death. The natural death term is simple, with $\mu_{B}{ }^{-1}$ being the mean life span of a seabird. Predation by cats is given by the predation rate $p_{B}$. Since cats tend to prey on seabirds only when there are few rabbits available, $p_{B}$ is divided by the total number of rabbits $(S+I+1)$.

Rabbits affect seabirds by destroying nesting sites, reducing seabird births. To eliminate the possibility of negative births, rabbits reduce seabird birth rates by division rather than subtraction. We assume the existence of refuges, nesting sites that rabbits cannot destroy; this prevents the birth rate from being reduced to 0 . The effect of new rabbits on births should decline as the number of rabbits increases, as undamaged nesting sites become harder to find. We then introduce $\Omega$, the maximum divisive effect of rabbits on number of seabird births and $k_{B}$, the logarithmic constant. Seabird birth rate is equal to $\Lambda$ when there are no rabbits $(S+I=0)$ and converges to $\Lambda / \Omega$ as the number of rabbits approaches infinity. Bringing together the seabird terms:

$$
\frac{d B}{d t}=\Lambda \div \Omega \frac{S+I+k_{B} / \Omega}{k_{B}+S+I}-\frac{p_{B} B C}{S+I+1}-\mu_{B} B
$$

Finally, we consider feral cats, which grow by consuming rabbits and seabirds and have a mean survival time of $\mu_{C}{ }^{-1}$. We ignore cat predation of mice and rats, which is less frequent and less nutritious than rabbit predation [4]. We introduce the terms $e_{R}$, the conversion efficiency (number of cats born per prey consumed) for consumed rabbits and $e_{B}$, the conversion efficiency for consumed birds. We assume susceptible and infected rabbits are equally nutritious. Predation on birds is mediated by the rabbit population: too few rabbits and cats will attack more birds. For example, when the rabbit population was severely reduced by myxomatosis in the 1980 s, the number of burrow-nesting seabird colonies dropped by almost $50 \%$ due to increased cat predation [4]. The cat eradication program that led to the extirpation of cats in 2000 did not begin in earnest until 1985, and so will not be explicitly inserted into the model (although the implications of predator removal will be considered). Bringing together the cat terms:

$$
\frac{d C}{d t}=e_{R} C\left(p_{S} S+p_{I} I\right)+e_{B} C\left(\frac{p_{B} B}{S+I+1}\right)-\mu_{C} C
$$

This leaves us with the full system of equations given in (2.9). The model can also be described visually using a state transition diagram, which shows the movement into, out of, and between classes (Figure 1). 


$$
\begin{aligned}
\frac{d B}{d t} & =\Lambda \div \Omega \frac{S+I+\frac{k_{B}}{\Omega}}{k_{B}+S+I}-\frac{p_{B} B C}{S+I+1}-\mu_{B} B \\
\frac{d S}{d t} & =\alpha S\left(1-\frac{S}{K}\right)-p_{S} S C-f(t) \beta S I-f(t) g(t) \sigma S \\
\frac{d I}{d t} & =f(t) \beta S I+f(t) g(t) \sigma S-p_{I} I C-\mu_{I} I \\
\frac{d C}{d t} & =e_{R} C\left(p_{S} S+p_{I} I\right)+e_{B} C\left(\frac{p_{B} B}{S+I+1}\right)-\mu_{C} C
\end{aligned}
$$

where $f(t)$ is a function defined: $f(t)=\frac{(1-r) / r}{(1-r) / r+e^{q t}}+r$

$$
\text { with } q=\frac{1}{x} \ln \left(\frac{1+r}{r}\right)
$$

where $g(t)$ is a function defined: $g(t)=-\left(\frac{1-\delta}{2}\right) \cos (2 \pi t)+\frac{1+\delta}{2}$

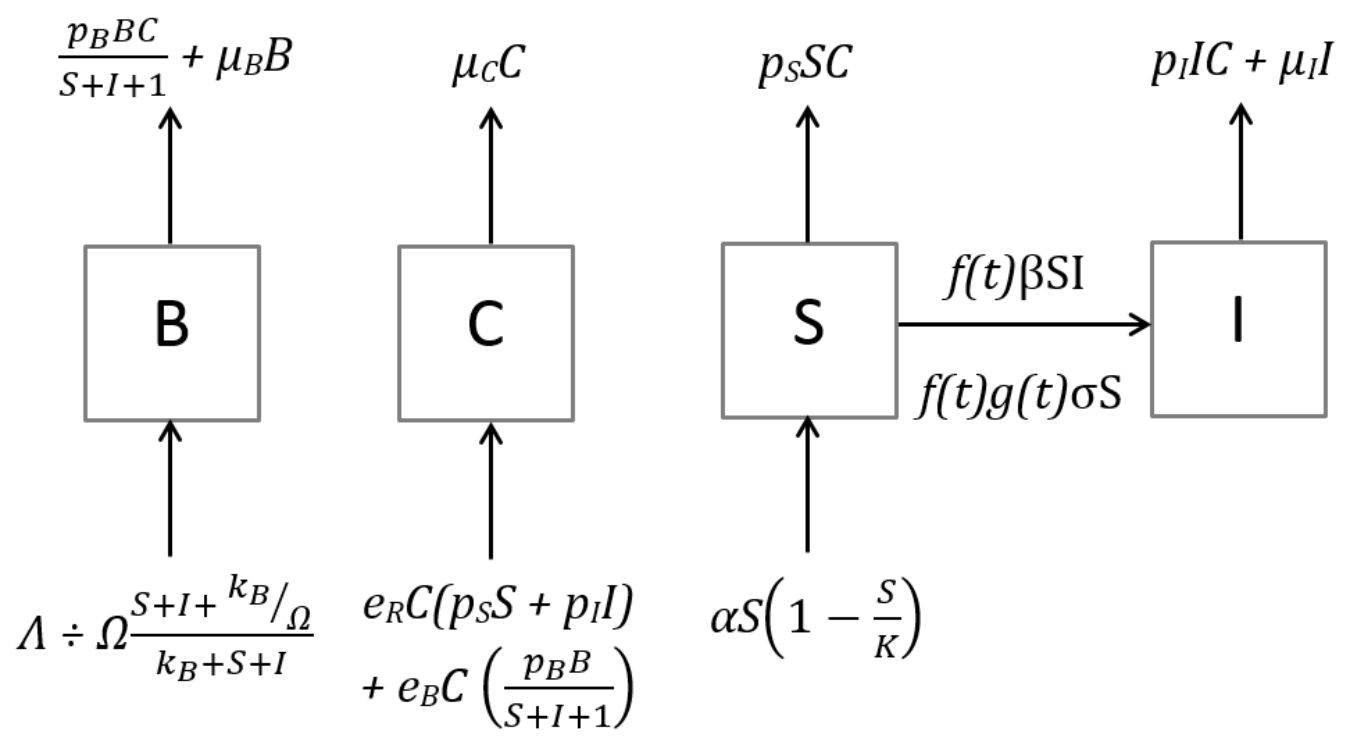

FIG. 1. State transition diagram of the model.

3. No Seabirds. The full model is too complex to examine analytically all at once. For example, we find that steady states where the predator exists (i.e. $C \neq 0$ ) are implicit. Thus we begin by removing the most complex element of the model: seabirds. We also ignore transmission by fleas, since a disease-free steady state is only possible in their absence. We analyse the following simplified model: 


$$
\begin{aligned}
\frac{d S}{d t} & =\alpha S\left(1-\frac{S}{K}\right)-p_{S} S C-f(t) \beta S I \\
\frac{d I}{d t} & =f(t) \beta S I-p_{I} I C-\mu_{I} I \\
\frac{d C}{d t} & =e_{R} C\left(p_{S} S+p_{I} I\right)-\mu_{C} C
\end{aligned}
$$

This model has four equilibria (ignoring the trivial case). We replace $f(t)$ with $r$, since $f(t)$ tends toward $r$ in the long-term.

$$
\begin{aligned}
S & =K \\
I & =0 \quad \text { (No disease, no predators) } \\
C & =0 \\
S & =\frac{\mu_{C}}{e_{R} p_{S}} \\
I & =0 \\
C & =\frac{\alpha}{p_{S}}\left(1-\frac{\mu_{C}}{K e_{R} p_{S}}\right) \quad \text { (No disease) } \\
S & =\frac{\mu_{I}}{r \beta} \\
I & =\frac{\alpha}{r \beta}\left(1-\frac{\mu_{I}}{K r \beta}\right) \quad \text { (No predators) } \\
C & =0
\end{aligned}
$$

$$
\begin{aligned}
& S=\frac{p_{I} C+\mu_{I}}{r \beta} \\
& I=\frac{\mu_{C}-e_{R} p_{S}\left(\frac{p_{I} C+\mu_{I}}{r \beta}\right)}{e_{R} p_{I}} \quad \text { (Endemic equilibrium with predators) } \\
& C=\frac{\alpha}{p_{S}}\left(1-\frac{p_{I} C+\mu_{I}}{K r \beta}\right)-\frac{r \beta\left(\mu_{C}-e_{R} p_{S}\left(\frac{p_{I} C+\mu_{I}}{r \beta}\right)\right)}{p_{S} e_{R} p_{I}}
\end{aligned}
$$

The Jacobian for this model is the following:

$$
\left(\begin{array}{ccc}
-C p_{S}-\alpha\left(\frac{S}{K}-1\right)-I \beta f(t)-\frac{S \alpha}{K} & -S \beta f(t) & -S p_{S} \\
I \beta f(t) & S \beta f(t)-p_{I} C-\mu_{I} & -p_{I} I \\
C e_{R} p_{S} & p_{I} C e_{R} & e_{R}\left(S p_{S}+p_{I} I\right)-\mu_{C}
\end{array}\right)
$$

The local stability of the four steady states can be assessed using the Jacobian. Unfortunately, the eigenvalues are far too complex to comment on for all but the simplest case. For the equilibrium with 0 cats and 0 infected rabbits:

$$
\lambda_{1}=K \psi r \psi \beta-\mu_{I}, \lambda_{2}=K \psi e_{R \psi} p_{S \psi}-\mu_{C}, \lambda_{3}=-\alpha \psi
$$


Thus the local stability of this equilibrium (i.e. long-term persistence or extirpation of cats and infected rabbits in the system) depends only upon $\lambda_{1}$ and $\lambda_{2}$ being negative, as $\lambda_{3}$ is always negative. This gives us two conditions for this equilibrium to be locally stable:

$$
\mu_{I}>K r \beta, \mu_{C}>K e_{R} p_{S}
$$

The death rate of infected rabbits must be greater than a function of the available pool of rabbits to be infected $(K$ and $f(t)$ ) and the speed at which they can be infected $(\beta)$. Also, the death rate of cats must be greater than a function of the available pool of prey $(K)$ and the ability to convert them into new cats $\left(e_{R}\right.$ and $\left.p_{S}\right)$. Interestingly, the growth rate of rabbits $(\alpha)$ is not present in either condition. As long as at least one of these conditions is not met, both disease and predators have the potential to persist long-term in a system without seabirds.

4. Rabbit Infection Dynamics. Next, we investigate the infection dynamics of rabbits and the effect of predatory cats on the disease. We will analyse increasingly complex versions of the infection model.

4.1. $R_{0}$ with no predators. We begin with the simplest possible set of equations, ignoring both predators and flea transmission, as the cyclical nature of the latter component would mean that the system has no true non-trivial steady states.

$$
\begin{aligned}
& \frac{d S}{d t}=\alpha S\left(1-\frac{S}{K}\right)-f(t) \beta S I \\
& \frac{d I}{d t}=f(t) \beta S I-\mu_{I} I
\end{aligned}
$$

This system has two equilibria; the disease-free equilibrium and the endemic equilibrium, respectively:

$$
\begin{array}{lll}
S=K & S=\frac{\mu_{I}}{r \beta} \\
I=0 & (4.2) & I=\frac{\alpha}{r \beta}\left(1-\frac{\mu_{I}}{K r \beta}\right)
\end{array}
$$

We use the next-generation method to calculate $R_{0}$ (see Appendix A for complete $R_{0}$ calculations). Remember that $f(t)=1$ initially:

$$
R_{0}=\frac{\beta K}{\mu_{I}}
$$

The disease is more likely to thrive with a higher contact rate $(\beta)$ and in a denser population $(K)$ and less likely to take hold with a higher disease mortality rate $\left(\mu_{I}\right)$. We can use PRCC analysis to determine which parameters (within a plausible range) exert the greatest influence on the $R_{0}$ for the disease (see Appendix $\mathbf{B}$ for a discussion of how parameter values were obtained). The disease parameters exert a larger influence on $R_{0}$ than carrying capacity, but a larger carrying capacity still has a positive effect on the probability that the disease will spread (Figure 2). These general observations are intuitive but do not capture what is interesting and unique about the Macquarie Island system: the interaction between disease and predators. 

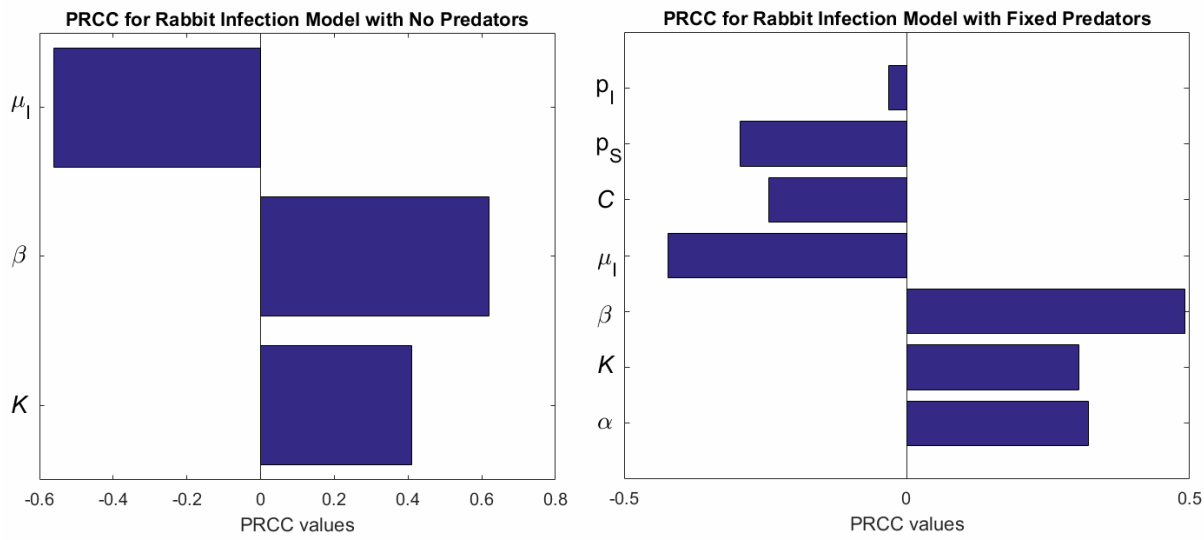

FIG. 2. Partial Rank Correlation Coefficient analysis using Latin Hypercube Sampling ( $n=$ 1000) for the rabbit infection dynamics model with no predators and fixed predators. See Appendix $C$ for parameter value ranges.

4.2. $R_{0}$ with fixed predators. We make our first attempt at incorporating predators by treating cats $(C)$ as a constant value.

The equations are as follows:

$$
\begin{aligned}
& \frac{d S}{d t}=\alpha S\left(1-\frac{S}{K}\right)-p_{S} S C-f(t) \beta S I \\
& \frac{d I}{d t}=f(t) \beta S I-p_{I} I C-\mu_{I} I
\end{aligned}
$$

The disease-free equilibrium and the endemic equilibrium, respectively:

$$
\begin{aligned}
& S=K\left(1-\frac{p_{S} C}{\alpha}\right) \\
& I=0
\end{aligned}
$$

$$
\begin{aligned}
& S=\frac{p_{I} C+\mu_{I}}{r \beta} \\
& I=\frac{\alpha\left(1-\frac{p_{I} C+\mu_{I}}{K r \beta}\right)-p_{S} C}{r \beta}
\end{aligned}
$$

The disease-free equilibrium is simple to understand. The rabbit population tends toward some proportion of the carrying capacity of the population $(K)$; this proportion is smaller when cats are more numerous $(C)$ and hunt more often $\left(p_{S}\right)$ and larger when the rabbit growth rate is high $(\alpha)$.

The endemic equilibrium is more interesting. First, we see that the populations of both susceptible and infected rabbits are dependent upon the susceptibility of rabbits to disease $(f(t)$, which begins at 1 and tends toward $r$ in the long-term) and the contact rate of the disease $(\beta)$. For susceptible rabbits, the lower the contact rate and proportion of susceptible rabbits, the higher the stable population of susceptible rabbits; with declining susceptibility over time, we expect to see more susceptible rabbits. Secondly, we see that the stable value of susceptible rabbits is independent of growth rate $(\alpha)$ and predation on susceptible rabbits $\left(p_{S}\right)$, and is instead determined by predation on infected rabbits $\left(p_{I}\right)$, the mortality rate of infected rabbits $\left(\mu_{I}\right)$, and the aforementioned disease parameters. The stable population of infected rabbits is determined by the growth rate, predation on infection and susceptible rabbits, the carrying capacity of susceptible rabbits $(K)$, and the two disease terms. 


$$
R_{0}=\frac{\beta K\left(1-\frac{p_{S} C}{\alpha}\right)}{p_{I} C+\mu_{I}}
$$

$R_{0}$ depends on every parameter except $r$ (which influences the number of infected rabbits in the long-term, as seen previously). $R_{0}$ is influenced positively by the contact rate $(\beta)$, the carrying capacity of susceptibles $(K)$, and the growth rate of rabbits $(\alpha) ; R_{0}$ is negatively influenced by predation on either form of rabbit $\left(C, p_{S}, p_{I}\right)$ and mortality caused by the disease $\left(\mu_{I}\right)$.

The two most important parameters are the contact rate $(\beta)$ and the mortality rate of infected rabbits $\left(\mu_{I}\right)$ (Figure 2). This makes sense because the diseased rabbits must live long enough and be sufficiently infectious for the disease to be able to take hold. We also see that the disease is more likely to spread when there is a large population of rabbits available to infect - that is, when the rabbit population is dense, fast-growing, and relatively unmolested by predators. The least important parameter is predation on infected rabbits, likely because the speed of infected rabbit death from disease greatly outpaces the speed of predation on diseased rabbits. Importantly, predation on susceptible rabbits matters much more.

We will see in the next section that including dynamic predators significantly changes the results. In this sense, we can view the fixed predators scenario as more applicable to a static human control measure, such as shooting a certain proportion of rabbits, rather than a dynamic, responsive biological control like the cats on Macquarie Island.

4.3. $R_{0}$ with dynamic predators. We re-add rate of change for cats and find the disease-free equilibrium:

$$
\begin{aligned}
& \frac{d S}{d t}=\alpha S\left(1-\frac{S}{K}\right)-f(t) \beta S I \\
& \frac{d I}{d t}=f(t) \beta S I-p_{I} I C-\mu_{I} I \\
& S=\frac{\mu_{C}}{e_{R} p_{S}} \\
& \frac{d C}{d t}=e_{R} C\left(p_{S} S+p_{I} I\right)-\mu_{C} C \\
& I=0 \\
& C=\frac{\alpha}{p_{S}}\left(1-\frac{\mu_{C}}{K e_{R} p_{S}}\right)
\end{aligned}
$$

$$
R_{0}=\frac{\beta \mu_{C}}{e_{R} p_{S}\left(\frac{p_{I} \alpha}{p_{S}}\left(1-\frac{\mu_{C}}{K e_{R} p_{S}}\right)+\mu_{I}\right)}
$$

Additionally, we test the effects of the assumption that rabbit growth is logistic by contrasting the logistic growth equation in (4.9) with the exponential growth equation in (4.12):

$$
\begin{aligned}
& \frac{d S}{d t}=\alpha S-p_{S} S C-f(t) \beta S I \\
& \frac{d I}{d t}=f(t) \beta S I-p_{I} I C-\mu_{I} I \\
& \frac{d C}{d t}=e_{R} C\left(p_{S} S+p_{I} I\right)-\mu_{C} C
\end{aligned}
$$

$$
\begin{aligned}
S & =\frac{\mu_{C}}{e_{R} p_{S}} \\
I & =0 \\
C & =\frac{\alpha}{p_{S}}
\end{aligned}
$$




$$
R_{0}=\frac{\beta \mu_{C} p_{S}}{e_{R} p_{S}\left(p_{I} \alpha+p_{S} \mu_{I}\right)}
$$
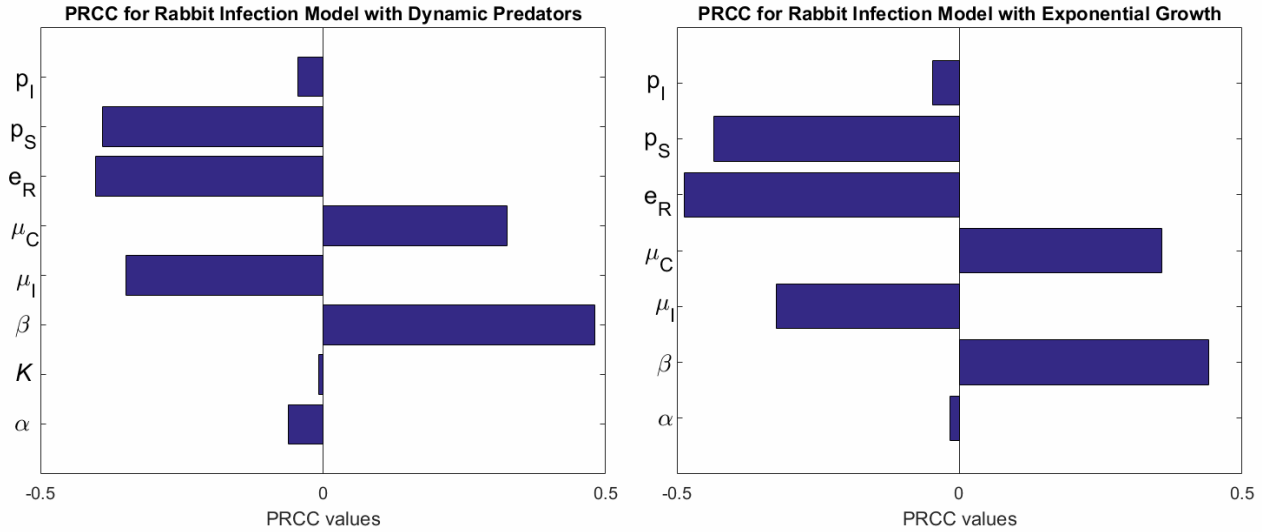

FIG. 3. Partial Rank Correlation Coefficient analysis using Latin Hypercube Sampling ( $n=$ 1000) for the rabbit infection dynamics model with dynamic predators and logistic or exponential growth. See Appendix $C$ for parameter value ranges.

We find that the growth rate $(\alpha)$ and carrying capacity $(K)$ of rabbits matter much less under these conditions, because the cat population is able to respond accordingly to the greater availability of prey (Figure 3). We still find $\beta$ and $\mu_{I}$ to be important parameters, but the cat parameters $\left(\mu_{C}, p_{S}, e_{R}\right.$ vs. $\left.C\right)$ are more important than they were in the fixed predator model. The predation rate on infected rabbits still contributes minimally to $R_{0}$.

With exponential growth, the results of PRCC analysis are very similar, indicating that dynamic predators are able to control the susceptible rabbits despite their less constrained growth in such a way that disease establishment is minimally affected (Figure 3). Thus the question of logistic versus exponential growth of the disease host with respect to disease establishment is not that important in a system where the host is controlled by a dynamic predator.

4.4. Myxomatosis and other diseases. The disease analysis leaves us with several conclusions:

- In general, diseases are more likely to be successful in denser, fast-growing populations. However, the presence of a predator whose growth is dependent on the disease host can mitigate the advantages to disease establishment of a fast-growing, densely-populated host. When the predator population is static in size (more akin to a human control measure, like hunting), the host population parameters maintain their importance.

- Increased predation reduces the chance that the disease will successfully establish. When predators are dynamic, predation parameters reduce the relative importance of disease parameters such as contact rate and infectious period to establishment.

- For a relatively fast-killing disease like myxomatosis, predation on infected individuals directly is much less important to disease establishment than predation on the supply of susceptible hosts. 
Myxomatosis is not the only disease that has been used to control rabbits. Like myxomatosis, rabbit haemorrhagic disease (RHD) was deliberately released to control the Europeans rabbit in New Zealand and Australia [10]. Similar to the initial introduction of myxomatosis, RHD has been brutally effective in killing rabbits; the first outbreak killed 14 million domesticated rabbits within 9 months in China [10]. Research suggests that RHD is most effective in certain situations and under certainly environmental conditions, particularly in denser rabbit populations (Story et al., 2004, as cited in [9]). How this density-dependant disease would interact with the presence of an abundant, effective predator remains to be investigated.

Theoretical research has also considered the interaction between RHD and myxomatosis. Although myxomatosis may suppress the prevalence of RHD during concurrent outbreaks, in other situations, myxomatosis may allow RHD to establish where it otherwise would not be able to [9]. Depending on conditions, it may be beneficial to simultaneously use multiple viruses as part of a biological pest control strategy.

4.5. Fleas and cats. Finally, we consider the addition of fleas to the rabbit infection model, with and without predators. As previously described, flea infection is described by a sinusoidal function to reflect the "unfavourable conditions" for Myxoma virus that required annual release:

$$
g(t)=-\left(\frac{1-\delta}{2}\right) \cos (2 \pi t)+\frac{1+\delta}{2}
$$

where the $\delta$ is the minimum effectiveness. The function is multiplied by the maximum flea contact rate, $\sigma$.

We can use a simplified model simulation (ignoring seabirds), to test the effect of flea addition with and without (i.e. $C_{0}=0$ ) predators:

$$
\begin{aligned}
\frac{d S}{d t} & =\alpha S\left(1-\frac{S}{K}\right)-p_{S} S C-f(t) \beta S I-f(t) g(t) \sigma S \\
\frac{d I}{d t} & =f(t) \beta S I+f(t) g(t) \sigma S-p_{I} I C-\mu_{I} I \\
\frac{d C}{d t} & =e_{R} C\left(p_{S} S+p_{I} I\right)-\mu_{C} C
\end{aligned}
$$

We make several observations from the simulations in Figure 4:

- Flea transmission increases seasonal variability in the susceptible rabbit population.

- In the presence of predators, the disease eventually becomes functionally extinct. This process takes longer in the presence of flea transmission.

- In the absence of predators, the disease can be maintained long-term. The presence of flea transmission causes oscillations to be sustained at a higher level.

Flea transmission causes greater seasonal variability in the population of susceptible rabbits. If the goal is protecting vegetation and seabird nesting sites, this property could be exploited in order to strategically reduce the rabbit population during the mating season for seabirds. Allowing the vegetation time to recover should improve the mating success of seabirds.

We also notice that the susceptible rabbit population actually drops following the functional extinction of the disease. In this case, releasing susceptible rabbits from the cycle of infection and quick death due to the disease gives the cats a functionally larger population to prey on, allowing their numbers to grow and forcing the rabbit 

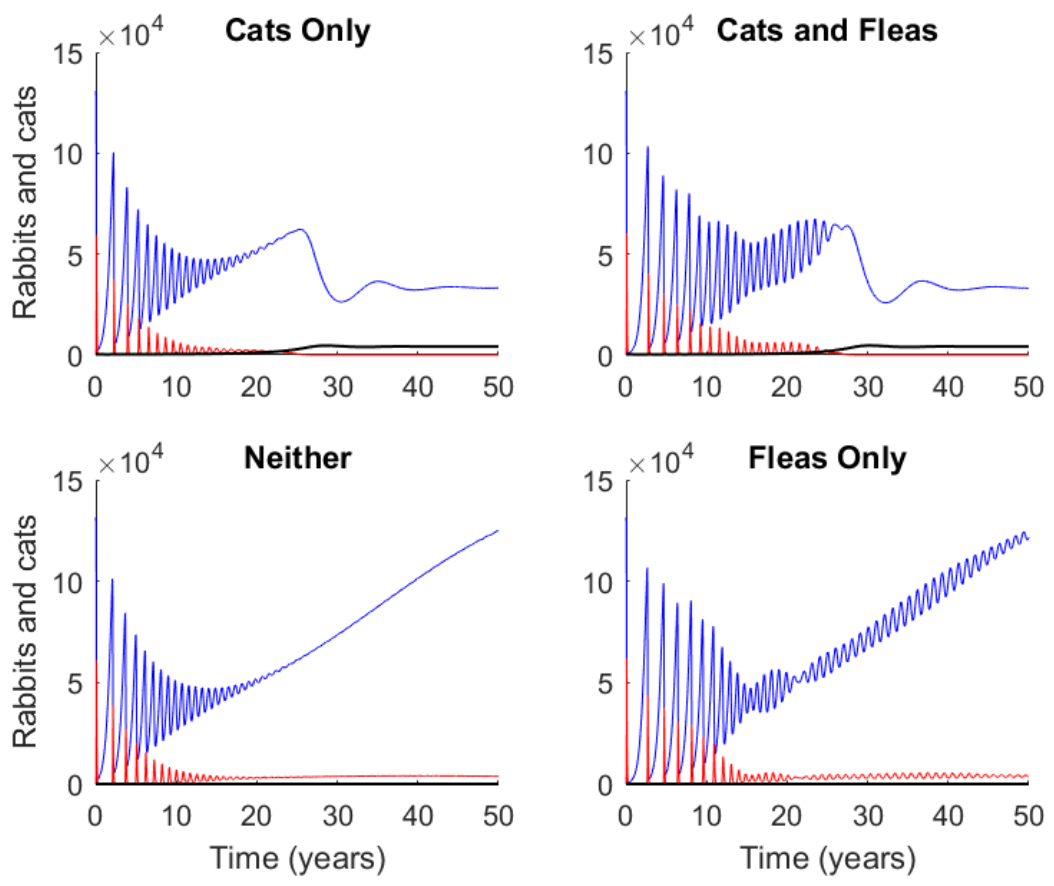

FIG. 4. 2x2 factorial series of numeric plots of the rabbit infection model with and without fleas and with and without predators. Susceptible rabbits (blue); infected rabbits (red); cats (black). See Appendix C for parameter values.

population toward a lower equilibrium. In the real Macquarie Island system, the virus release program was compromised around the same time the last feral cat on the island was shot (in 2000), so the above scenario was never played out in the real world. Still, this is a potential consideration for future pest control efforts.

5. Seabirds and Rabbits. To investigate the interaction between seabirds and rabbits, we ignore predation and disease and add a natural mortality term for rabbits, $\mu_{S}$. Equations (5.1) and (5.2) describe the system and its equilibrium:

$$
\begin{aligned}
& \frac{d B}{d t}=\Lambda \div \Omega \frac{S+I+\frac{k_{B}}{\Omega}}{k_{B}+S+I}-\mu_{B} B \\
& \frac{d S}{d t}=\alpha S\left(1-\frac{S}{K}\right)-\mu_{S} S \\
& B=\frac{\Lambda}{\mu_{B}} \div\left(\Omega \frac{K\left(1-\frac{\mu_{S}}{\alpha}\right)+\frac{k_{B}}{\Omega}}{k_{B}+K\left(1-\frac{\mu_{S}}{\alpha}\right)}\right) \\
& S=K\left(1-\frac{\mu_{S}}{\alpha}\right)
\end{aligned}
$$

The absence of predators is a realistic scenario, as the explosion of rabbits and subsequent decline of seabird nesting sites following the elimination of feral cats on Macquarie Island in 2000 (and apparent widespread resistance to myxomatosis) is what prompted the creation of this model. We can plot the equilibrium number of seabirds as a function of the carrying capacity of rabbits in both scenarios. 


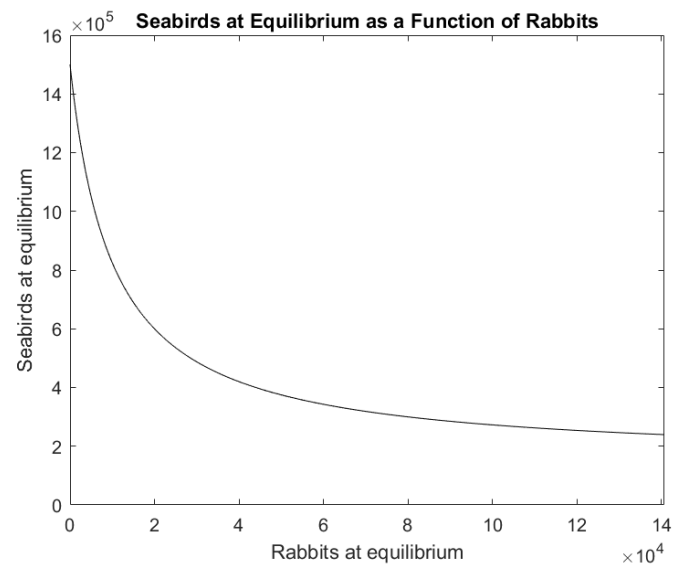

FIG. 5. Numeric plot of the equilibrium value of seabirds as a function of the equilibrium value of rabbits $\left(K\left(1-\frac{\mu_{S}}{\alpha}\right), K=0-200,000\right)$. See Appendix $C$ for parameter values.

In the absence of predators and disease, rabbit populations explode and seabirds crash. Since the reduction in seabird births by rabbits is logarithmic, a great reduction in rabbits is required to see a large benefit to seabirds (Figure 5). These kinds of shifts are possible, at least temporarily, as the initial introduction of myxomatosis reduced the rabbit population precipitously; the rabbit population went from 130,000 before release (in 1978) to a minimum of approximately 5,000-10,000 in 1988 [1,25]. However, these dramatic shifts in prey populations can have unintended consequences, as demonstrated in the next section.

6. Simulations. Simulations of the full model allow us to integrate what we have learned and ask more questions.

6.1. Disease versus predators. In the real Macquarie Island system, seabirds suffered greatly after the extirpation of cats due to the explosion of rabbits. In the following plot, we compare the outcome with respect to the seabird population of having only a single control measure: disease (with flea transmission) or predators.

As shown in Figure 6, the contact rate of the disease $(\beta)$ must be tripled in order to achieve the same long-term population of seabirds as in the system with only predators. Although cats are certainly harmful to seabirds, they may represent the lesser of two evils by helping to keep other pests under control. A "trophic cascade" is a phenomenon in food webs where a change in abundance in one level of the system has indirect effects on other levels. For example, the removal of a top predator releases lower predators from predation, potentially allowing their populations to increase. This may in turn cause lower-down prey species to suffer in their populations sizes. Although rabbits are not strictly "predators", they act like them by negatively influencing seabird reproduction. On Macquarie Island and similar islands, the elimination of top predators can have the opposite effect on conservation goals than intended by "releasing" species lower down on the food chain [1, 22].

It should be noted, however, that the shape of the seabird decline is significantly different under the disease-only scenarios versus the predator-only scenario. Even if the long-term stable value for seabird populations is the same or less in the diseaseonly scenarios, the actual number of seabirds is significantly higher for the first 50 or so years compared to the predator-only scenario, where the bird decline occurs 

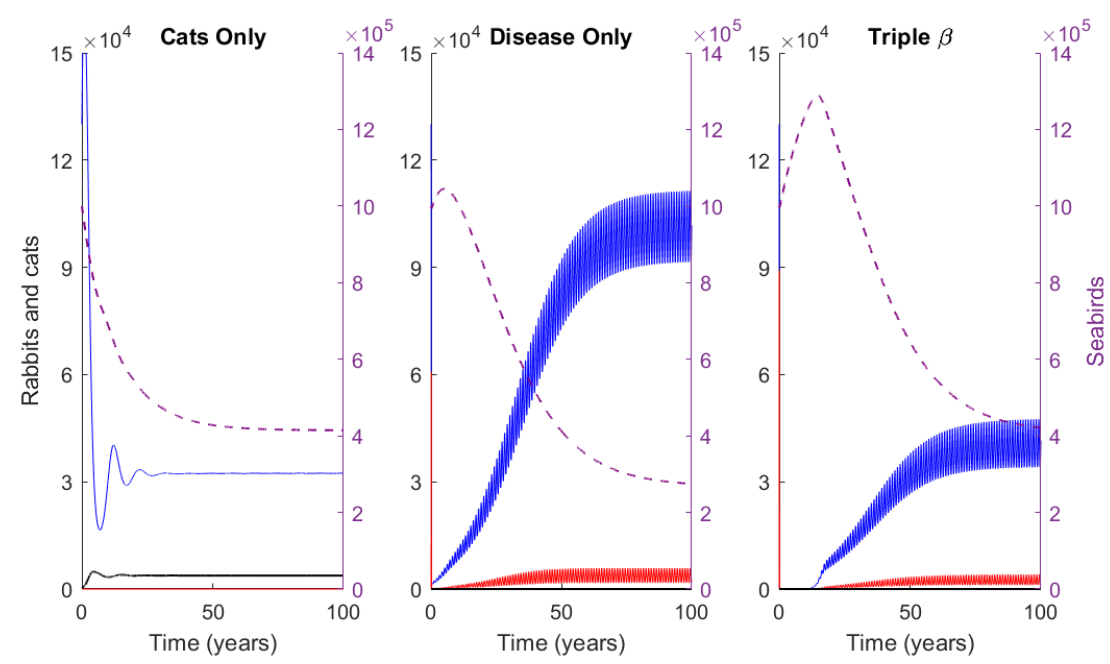

FIG. 6. Series of numeric plots of the Macquarie Island model with three different parameter combinations: no disease (1); no predators (2); no predators and triple $\beta$ (3). Susceptible rabbits (blue); infected rabbits (red); cats (black); seabirds (purple, dashed). See Appendix $C$ for parameter values.

much earlier. If more complex birth dynamics for seabirds were incorporated into the model, this property could become very important.

6.2. Full model and carrying capacity. Finally, we come to a simulation of the full model — with seabirds, disease, and predators.
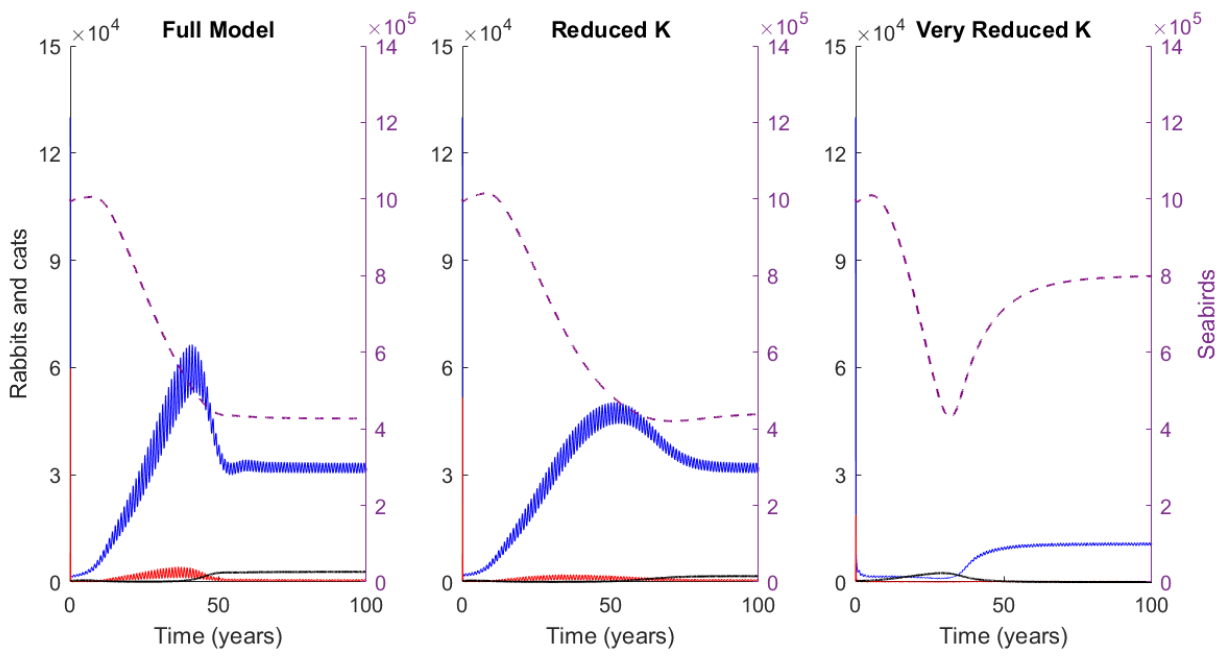

FIG. 7. Series of numeric plots of the entire Macquarie Island model with three different parameter combinations: $K \psi=200,000$ (1); $K \psi=75,000$ (2); $K \psi=12,940$ (3). Susceptible rabbits (blue); infected rabbits (red); cats (black); seabirds (purple, dashed). See Appendix $C$ for parameter values.

We see from the first plot in Figure 7 that having both predators and disease could give us the best of both worlds for each control measure. We have the same 
higher long-term outcome for seabirds as in the predator-only scenario and a similar delayed reduction of seabirds in the first 50 years as in the disease-only scenario. In practical terms, this favours simultaneous elimination of both pests, as having one without the other may do more damage than having both. In the disease modelling section, we identified carrying capacity $(K)$ as a relatively unimportant parameter for disease establishment. In these simulations, we find that the long-term number of seabirds (and even the general shape of the curve) is somewhat robust to changes in $K$, although changing $K$ does affect how the equilibrium is achieved. In the middle plot, there are fewer predators and fewer diseased rabbits - but the lower carrying capacity means that the number of susceptible rabbits ends up being similar, along with the seabirds. In the final plot, with a very reduced $K$, cats decimate seabird populations before going extinct due to a lack of rabbits. Historically, the introduction of a deadly disease like myxomatosis has shown the capability to produce sudden, prolonged plunges in the host population, highlighting the delicate balance between cats, rabbits, and seabirds. While it is unlikely that a drastically reduced effective carrying capacity of rabbits could be sustained over a long period of time, there may be significant short-term consequences for cats and seabirds.

7. Conclusions. The most challenging component of this system to model was also the most critical: the seabirds, the target of conservation. In the real Macquarie Island system, the unintended consequences on seabirds of eliminating cats were similarly difficult to foresee. This highlights the issues facing agencies in charge of making conservation and management decisions affecting a complex web of ecosystem interactions. Owing to the assumptions and compromises necessarily made as part of the modelling process, this Macquarie Island model is certainly flawed, and as a result may be unable to capture the true dynamics of the system, potentially in important ways. For example, the different species of seabird should be modelled individually, and their growth equations should be more reflective of their individual life history traits and mating patterns. Some parameters were more difficult to estimate than others. Additionally, other pest species like rats, which interact with both cats and seabirds, could be included.

The great statistician George Box said that "all models are wrong, but some are useful." Despite the limitations of the model, the various analyses undertaken revealed interesting qualitative trends that could inform future conservation efforts in complex predator-prey ecosystems, especially when considering the use of an infectious disease. The model demonstrated that the most important parameters to disease establishment changed depending on the nature of the other controls on the rabbit population. Unsurprisingly, contact rate $(\beta)$ and mean infectious period $\left(\mu_{I}^{-1}\right)$ were consistently very important parameters in the disease model. When combined with a static, nondisease control (e.g. hunting by humans), carrying capacity $(K)$ and growth rate $(\alpha)$ of the host population were also important; however, they were much less so in the presence of a dynamic predator (e.g. feral cats). With a dynamic predator, predation on the susceptible host population (but not infected hosts) became very important. Whether the hosts grew logistically or exponentially made little difference in this situation.

Analysis of seabirds and rabbits showed that reductions in rabbits must be substantial in order to provide the maximum benefit to seabird populations. Disease, predators, or some combination thereof can be used to this end. Simulations showed that these forces can interact in positive or negative ways. Predators can suppress disease spread and vice versa, but these two can also act in conjunction to slow the 
decline of seabird populations. Seasonal vector-based transmission of a disease may be particularly useful when the seasonal breeding patterns of seabirds are accounted for.

Ultimately, the point is moot for Macquarie Island in particular. Although the extermination of cats from the island was initially disastrous, recent intensive efforts to eliminate rabbits, mice, and rats have proved successful, leading to a remarkable recovery for seabirds [19]. But the fraught ecological history of Macquarie Island, along with the results of this paper, should motivate future conservation efforts to thoughtfully consider the potential indirect consequences of management decisions on the ecosystem as a whole. In closing, we present a short list of important considerations for ecosystem managers who find themselves in a similar position to those in charge of Macquarie Island in 1978:

- If the conservation goal is the protection of a particular species or groups of species, identify the organism(s) that influence their abundance. If there is more than one, identify how these organisms interact with each other, as well. There may be unintended consequences on other organisms (and eventually on the species to be protected) from control measures targeting a particular organism (e.g. trophic cascades).

- When considering a disease to control an organism, identify the characteristics of the disease that are most relevant to its successful establishment in the ecosystem at hand. If the host organism is uncontrolled by a predator, a disease that thrives in a densely-populated or fast-growing host may be preferable; these characteristics are less important if the host organism is also controlled by a predator. The presence of a predator may have serious implications on a disease's ability to establish and its long-term viability.

- Consider the effects of a disease on the potential host's predator(s), as well as other organisms interacting with it. Depending on conditions, the disease may indirectly influence the predator's population size or the primary target of its predation (which may become the species intended to be conserved). Changes in the population size of the predator may have long-term impacts on the predator's ability to control the host organism independent of the disease.

- Some predators control other predators. While these top predators may do harm to the target of conservation, eliminating them may cause the growth of some of their prey species to become unconstrained. These species may have the potential to do even greater harm to the conservation effort. 


\section{Appendix A: $R_{0}$ calculations.}

The basic reproductive ratio $\left(R_{0}\right)$ describes the average number of secondary cases produced by a single infectious individual over their infectious lifetime in a completely susceptible population. $R_{0}$ acts as a threshold wherein a disease is able to invade an uninfected population if $R_{0}>1$ and is unable to invade if $R_{0}<1$.

The calculate $R_{0}$ for each version of the model using the next-generation method [7]. In short, the next-generation begins by defining the vector $\bar{x}=x_{i}$, where $x_{i}$ gives the number of individuals in the $i$ th infective class. In our model, there is only a single infective class, $I$. Next, we define $F_{i}(\bar{x})$ as the rate of new infections in the $i$ th class from the susceptible class (in our model, $S$ ). Finally, we define $V_{i}(\bar{x})=V_{i}^{-}(\bar{x})-V_{i}^{+}(\bar{x})$, where $V_{i}^{+}$is the transfer of individuals into the $i$ th infective class from all other classes excluding the susceptible class and $V_{i}^{-}$is the transfer of individuals out of the $i$ th infective class.

$$
F=\left[\frac{\partial F_{i}\left(x_{0}\right)}{\partial x_{j}}\right], \quad V=\left[\frac{\partial V_{i}\left(x_{0}\right)}{\partial x_{j}}\right]
$$

The value for $R_{0}$ is given as the dominant eigenvalue of the matrix $F V^{-1}$.

$R_{0}$ with no predators.

$$
\begin{gathered}
F=[f(t) \beta S]=[\beta K], V^{-1}=\left[\frac{1}{\mu_{I}}\right] \\
F V^{-1}=R_{0}=\frac{\beta K}{\mu_{I}}
\end{gathered}
$$

$R_{0}$ depends on the rabbit disease susceptibility function $(f(t))$, the contact rate of an infected rabbit $(\beta)$, the number of susceptible rabbits $(S)$, the carrying capacity of the rabbit population $(K)$, and the mortality rate of infected rabbits $\left(\mu_{I}\right)$.

$R_{0}$ with fixed predators.

$$
\begin{gathered}
F=[f(t) \beta S]=\left[\beta K\left(1-\frac{p_{S} C}{\alpha}\right)\right], V^{-1}=\left[\frac{1}{p_{I} C+\mu_{I}}\right] \\
F V^{-1}=R_{0}=\frac{\beta K\left(1-\frac{p_{S} C}{\alpha}\right)}{p_{I} C+\mu_{I}}
\end{gathered}
$$

$R_{0}$ depends on the predation rate on susceptible rabbits $\left(p_{S}\right)$, the number of cats $(C)$, the maximum per capita growth rate per susceptible rabbit $(\alpha)$, the predation rate on infected rabbits $\left(p_{I}\right)$, and terms already mentioned above.

\section{$R_{0}$ with dynamic predators.}

$$
\begin{gathered}
F=[f(t) \beta S]=\left[\frac{\beta \mu_{C}}{e_{R} p_{S}}\right], V^{-1}=\left[\frac{1}{p_{I} C+\mu_{I}}\right]=\left[\frac{1}{\frac{p_{I} \alpha}{p_{S}}\left(1-\frac{\mu_{C}}{K e_{R} p_{S}}\right)+\mu_{I}}\right] \\
F V^{-1}=R_{0}=\frac{\beta \mu_{C}}{e_{R} p_{S}\left(\frac{p_{I} \alpha}{p_{S}}\left(1-\frac{\mu_{C}}{K e_{R} p_{S}}\right)+\mu_{I}\right)}
\end{gathered}
$$

$R_{0}$ depends on the mortality rate of cats $\left(\mu_{C}\right)$, the rabbit conversion efficiency $\left(e_{R}\right)$, and terms already mentioned above. 
$R_{0}$ with exponential growth.

$$
\begin{gathered}
F=[f(t) \beta S]=\left[\frac{\beta \mu_{C}}{e_{R} p_{S}}\right], V^{-1}=\left[\frac{1}{p_{I} C+\mu_{I}}\right]=\left[\frac{p_{S}}{p_{I} \alpha+p_{S} \mu_{I}}\right] \\
F V^{-1}=R_{0}=\frac{\beta \mu_{C} p_{S}}{e_{R} p_{S}\left(p_{I} \alpha+p_{S} \mu_{I}\right)}
\end{gathered}
$$

$R_{0}$ depends on terms already mentioned above.

\section{Appendix B: Parameter values \& initial conditions.}

A great many sources were used to estimate parameter values and initial condi-

\begin{tabular}{|c|c|}
\hline Symbol & Sources \\
\hline$B_{0}$ & $500,000-1,500,000$ \\
\hline \multicolumn{2}{|c|}{ Initial number of seabirds (birds) } \\
\hline \multicolumn{2}{|c|}{$\begin{array}{l}\text { The main breeding seabirds on Macquarie Island are penguins. Petrels are also important } \\
\text { birds. Approximately } 3.5 \text { million seabirds visit the island annually to breed and moult. } \\
\text { Many of these birds do not stay on the island year-round. The most populous bird, the } \\
\text { Royal Penguin, is estimated at } 1.7 \text { million and stay on the island for seven months of the } \\
\text { year, whereas } 200,000 \text { King Penguins stay all year. Some birds may be too large for cats to } \\
\text { effectively hunt. We estimate the number of birds available to interact with the Macquarie } \\
\text { Island system to be between } 500,000 \text { and } 1.5 \text { million. }\end{array}$} \\
\hline$S_{0}$ & $130,000 \quad[1]$ \\
\hline \multicolumn{2}{|c|}{ Initial number of susceptible rabbits (rabbits) } \\
\hline \multicolumn{2}{|c|}{ The rabbit population just before virus release in 1978 . } \\
\hline$I_{0}$ & 100 \\
\hline \multicolumn{2}{|c|}{ Initial number of infected rabbits (rabbits) } \\
\hline \multicolumn{2}{|c|}{ We begin with a small population of infected rabbits. } \\
\hline$C_{0}$ & $350-550$ \\
\hline \multicolumn{2}{|r|}{ Initial number of cats (cats) } \\
\hline \multicolumn{2}{|c|}{$\begin{array}{l}\text { Based on the eradication program described in [1], we can assume a cat population any- } \\
\text { where from } 300-550 \text {. }\end{array}$} \\
\hline$\Lambda$ & $\begin{array}{lc}75,000-125,000 & {[18] ;[16] ;[3]} \\
\end{array}$ \\
\hline \multicolumn{2}{|r|}{ Constant birth rate of seabirds (birds) } \\
\hline \multicolumn{2}{|c|}{$\begin{array}{l}\text { Some seabirds breed every year, some do not. A King Penguin produces only a single } \\
\text { egg per year while breeding (although breeding is not always successful) [3]. Other birds } \\
\text { produce more eggs, so the base number of new birds every year is difficult to estimate. } \\
\text { A conservative estimate of breeding pairs from [16] would put the number of new birds } \\
\text { each year at approximately } 150,000 \text {; however, not all of these birds would be be eligible to } \\
\text { interact with the model. Also, not all birds would survive to maturity. }\end{array}$} \\
\hline
\end{tabular}
tions. Simulations were also used in some cases to help determine reasonable values. 


\begin{tabular}{|c|c|}
\hline & $0.5-5.5 \quad[1] ;$ Skira (1978) as cited in [8]; [26] \\
\hline \multicolumn{2}{|r|}{ Maximum per capita growth rate per susceptible rabbit (years $\left.{ }^{-1}\right)$} \\
\hline \multicolumn{2}{|c|}{$\begin{array}{l}\text { Skira (1978) reported } 19.3 \text { rabbit kittens per breeding female per year during the } 1974-1975 \\
\text { breeding season, with as many as } 57 \% \text { of females being pregnant in a season. This suggests } \\
\text { a per capita birth rate of } 5.5 \text { (remember, only half of the population is reproductive); } \\
\text { however, high early mortality must also be taken into account [26]. Cats were eliminated } \\
\text { in } 2000 \text { and the rabbit population increased from approximately } 10,000 \text { in that year to an } \\
\text { estimated } 130,000 \text { in } 2006 \text { [1]. Assuming logistic growth with } \mathrm{K}=187,500 \text { in the absence of } \\
\text { cats and with a largely ineffective virus, growth over six years suggests } \alpha=0.62 \text {. Of course, } \\
\text { we must also remember that only half of the rabbit population (females) is reproductive. }\end{array}$} \\
\hline$K$ & $150,000-225,000 \quad[24] ;$ Terauds (2009) as cited in [21] \\
\hline \multicolumn{2}{|c|}{ Carrying capacity of rabbit population (rabbits) } \\
\hline \multicolumn{2}{|r|}{$\begin{array}{l}\text { The rabbit population was estimated at } 150,000 \text { in } 1966 \text { (before Myxomatosis but with cat } \\
\text { predation). Estimates of the rabbit population in } 1978 \text { right before virus release range from } \\
130,000-150,000[1,4] \text {. Cats were eliminated in } 2000 \text { and the rabbit population increased } \\
\text { from approximately } 10,000 \text { in that year to an estimated } 130,000 \text { in } 2006 \text { [1]. We use a } \\
\text { range of } 150,000 \text { to } 50 \% \text { more in the absence of cats. }\end{array}$} \\
\hline$\beta$ & \begin{tabular}{|cc}
$0.0001-0.01+[9]$
\end{tabular} \\
\hline \multicolumn{2}{|c|}{ Contact rate of an infected rabbit $\left(\right.$ rabbits $^{-1}$ years $\left.^{-1}\right)$} \\
\hline \multicolumn{2}{|r|}{$\begin{array}{l}\text { Investigating this value will be a key component of the analysis. [9] references a per-hectare } \\
\text { contact rate for myxomatosis. }\end{array}$} \\
\hline & $\begin{array}{ll}0.5-3 & {[17]}\end{array}$ \\
\hline \multicolumn{2}{|c|}{ Maximum effectiveness of flea transmission $\left(\right.$ years $\left.^{-1}\right)$} \\
\hline \multicolumn{2}{|r|}{$\begin{array}{l}\text { It is difficult to estimate the relative effectiveness of fleas versus other rabbits in the spread } \\
\text { of myxomatosis. [17] reports on the release program. }\end{array}$} \\
\hline & 0.25 \\
\hline \multicolumn{2}{|c|}{ Minimum effectiveness of flea transmission (proportion of max.) (dimensionless) } \\
\hline \multicolumn{2}{|r|}{ We set minimum flea effectiveness to $25 \%$ of maximum. } \\
\hline & 10 \\
\hline \multicolumn{2}{|r|}{ Maximum decrease in seabird births by rabbits (by division) (dimensionless) } \\
\hline \multicolumn{2}{|r|}{$\begin{array}{l}\text { This value is difficult to estimate. We assume rabbits can reduce bird births by an order } \\
\text { of magnitude at maximum. See } k_{B} \text { for how this reduction works in practice. }\end{array}$} \\
\hline & \begin{tabular}{|lc}
$1 / 20-1 / 15$ & {$[20] ;[5]$}
\end{tabular} \\
\hline \multicolumn{2}{|c|}{ Mortality rate of seabirds (years ${ }^{-1}$ ) } \\
\hline \multicolumn{2}{|r|}{$\begin{array}{l}\text { Some of the birds on Macquarie Island are relatively long-lived: e.g. King Penguin (15-20 } \\
\text { years) and Southern Giant Petrel (some live more than } 30 \text { years). We take this value to } \\
\text { be } 15-20 \text { years. }\end{array}$} \\
\hline & $365 / 12-365 / 10 \quad[12]$ \\
\hline \multicolumn{2}{|c|}{ Mortality rate of infected rabbits (years ${ }^{-1}$ ) } \\
\hline \multicolumn{2}{|c|}{ The average survival time for non-attenuated strains is 10-12 days. } \\
\hline & AVMA (2003) as cited in [11] \\
\hline \multicolumn{2}{|c|}{ Mortality rate of cats $\left(\right.$ years $^{-1}$ ) } \\
\hline & ted. We vary $\mathrm{t}$ \\
\hline
\end{tabular}




\begin{tabular}{|c|c|}
\hline \multirow{2}{*}{\multicolumn{2}{|c|}{\begin{tabular}{l|l}
$p_{B}$ & $0.025-0.1$ \\
Predation rate on seabirds (rabbits cats & -1 \\
years & -1
\end{tabular}}} \\
\hline & \\
\hline \multicolumn{2}{|c|}{$\begin{array}{l}\text { In the } 1970 \text { s, cats were killing } 60,000 \text { burrowing seabirds a year. Given that abundant } \\
\text { rabbits suppress cat predation of birds, then cat predation of birds would be much higher } \\
\text { in the absence of rabbits. Since there were only several hundred cats at this time, we might } \\
\text { expect that the base predation rate would be on the order of } 1000 \text { birds per cat per year. }\end{array}$} \\
\hline$p_{S}$ & $0.00025-0.00075 \quad[1]$ \\
\hline \multicolumn{2}{|r|}{ Predation rate on susceptible rabbits $\left(\mathrm{cats}^{-1}\right.$ years $\left.^{-1}\right)$} \\
\hline \multicolumn{2}{|c|}{$\begin{array}{l}\text { [1] conservatively estimate that the } 157 \text { cats shot in } 1997 \text { consumed at least } 4000 \text { adult- } \\
\text { sized rabbits per year, or the equivalent of up to } 13,700 \text { rabbit kittens per year. This places } \\
\text { the number of rabbits consumed per cat per year at } 25 \text { or greater. }\end{array}$} \\
\hline$p_{I}$ & $0.00025-0.00075 \quad[1]$ \\
\hline \multicolumn{2}{|c|}{ Predation rate on infected rabbits $\left(\mathrm{cats}^{-1}\right.$ years $\left.{ }^{-1}\right)$} \\
\hline \multicolumn{2}{|c|}{$\begin{array}{l}\text { Although infirm rabbits may be easier to catch than healthy rabbits, they may also be less } \\
\text { likely to leave their burrows, so we will assume that } p_{I} \text { is roughly equal to } p_{S} \text {. }\end{array}$} \\
\hline$e_{B}$ & $0.0075 \quad[17]$ \\
\hline \multicolumn{2}{|r|}{ Seabird conversion efficiency (cats born per seabird consumed) (cats birds ${ }^{-1}$ ) } \\
\hline \multicolumn{2}{|c|}{$\begin{array}{l}\text { See } p_{B}, e_{R}, p_{S} \text {; these suggest } e_{B}<e_{R} \text {, at least for the seabirds that most frequently } \\
\text { became the cat's prey. }\end{array}$} \\
\hline & $0.015 \quad$ Copson (2002) as cited in [1] \\
\hline \multicolumn{2}{|r|}{ Rabbit conversion efficiency (cats born per rabbit consumed) (cats rabbits ${ }^{-1}$ ) } \\
\hline \multicolumn{2}{|c|}{$\begin{array}{l}\text { Copson }(2002) \text { estimated cat recruitment rate at } 124 \text { cats/year between } 1985-1995 \text {, equal } \\
\text { to the kill rate of the eradication program. Based about what we know about the predation } \\
\text { rate of cats on rabbits, this suggests a conversion efficiency in the neighbourhood of } 0.015 \text {. }\end{array}$} \\
\hline & $0.2 \quad[12]$ \\
\hline \multicolumn{2}{|r|}{ Long-term proportion of rabbits susceptible to disease (dimensionless) } \\
\hline \multicolumn{2}{|r|}{$\begin{array}{l}\text { Reported fatality rates in various natural systems suggest the long-term fatality rate may } \\
\text { be around } 20 \% \text {. }\end{array}$} \\
\hline & $\begin{array}{lc}20 & {[12] ;[8]}\end{array}$ \\
\hline \multicolumn{2}{|c|}{ Time for rabbit susceptibility to disease to decay by half (years) } \\
\hline \multicolumn{2}{|r|}{$\begin{array}{l}{[12] \text { records significant resistance and attenuation arising in the first decade after release in }} \\
\text { other locations. [8] notes apparent widespread resistance to Myxoma on Macquarie Island } \\
\text { after } 26 \text { years. }\end{array}$} \\
\hline$k_{B}$ & 100,000 \\
\hline & \\
\hline
\end{tabular}

\section{Appendix C: Parameter values tested.}

\section{Rabbit Infection Dynamics}

$R_{0}$ with fixed predators

\begin{tabular}{|c|ll|}
\hline Symbol & Definition & Parameter range \\
\hline$K$ & Carrying capacity of rabbit population & $50,000-300,000$ \\
\hline$\beta$ & Contact rate of an infected rabbit & $0.0001-0.01$ \\
\hline$\mu_{I}$ & Mortality rate of infected rabbits & $365 / 50^{-365 / 2}$ \\
\hline
\end{tabular}


$R_{0}$ with fixed predators

\begin{tabular}{|c|ll|}
\hline Symbol & Definition & Parameter range \\
\hline$\alpha$ & Maximum per capita growth rate per susceptible rabbit & $0.5-10$ \\
\hline$K$ & Carrying capacity of rabbit population & $50,000-300,000$ \\
\hline$\beta$ & Contact rate of an infected rabbit & $0.0001-0.01$ \\
\hline$\mu_{I}$ & Mortality rate of infected rabbits & $365 / 50^{-365 / 2}$ \\
\hline$C$ & Constant number of cats & $50-1000$ \\
\hline$p_{S}$ & Predation rate on susceptible rabbits & $0.00005-0.005$ \\
\hline$p_{I}$ & Predation rate on infected rabbits & $0.00005-0.005$ \\
\hline
\end{tabular}

$\underline{R_{0} \text { with dynamic predators }}$

\begin{tabular}{|c|ll|}
\hline Symbol & Definition & Parameter range \\
\hline$\alpha$ & Maximum per capita growth rate per susceptible rabbit & $0.5-10$ \\
\hline$K$ & Carrying capacity of rabbit population & $50,000-300,000$ \\
\hline$\beta$ & Contact rate of an infected rabbit & $0.0001-0.01$ \\
\hline$\mu_{I}$ & Mortality rate of infected rabbits & $365 / 50^{-365 / 2}$ \\
\hline$\mu_{C}$ & Mortality rate of cats & $1 / 12-1$ \\
\hline$e_{R}$ & Rabbit conversion efficiency & $0.15-0.0015$ \\
\hline$p_{S}$ & Predation rate on susceptible rabbits & $0.00005-0.005$ \\
\hline$p_{I}$ & Predation rate on infected rabbits & $0.00005-0.005$ \\
\hline
\end{tabular}

$\underline{R_{0} \text { with exponential rabbit growth }}$

\begin{tabular}{|c|ll|}
\hline Symbol & Definition & Parameter range \\
\hline$\alpha$ & Maximum per capita growth rate per susceptible rabbit & $0.5-10$ \\
\hline$\beta$ & Contact rate of an infected rabbit & $0.0001-0.01$ \\
\hline$\mu_{I}$ & Mortality rate of infected rabbits & $365 / 50^{-365 / 2}$ \\
\hline$\mu_{C}$ & Mortality rate of cats & $1 / 12-1$ \\
\hline$e_{R}$ & Rabbit conversion efficiency & $0.15-0.0015$ \\
\hline$p_{S}$ & Predation rate on susceptible rabbits & $0.00005-0.005$ \\
\hline$p_{I}$ & Predation rate on infected rabbits & $0.00005-0.005$ \\
\hline
\end{tabular}

Fleas and cats

\begin{tabular}{|c|ll|}
\hline Symbol & Definition & Value \\
\hline$S_{0}$ & Initial number of susceptible rabbits & 130,000 \\
\hline$I_{0}$ & Initial number of infected rabbits & 100 \\
\hline$C_{0}$ & Initial number of cats & 0,350 \\
\hline$\alpha$ & Maximum per capita growth rate per susceptible rabbit & 2.5 \\
\hline$K$ & Carrying capacity of rabbit population & 200,000 \\
\hline$\beta$ & Contact rate of an infected rabbit & 0.001 \\
\hline$\sigma$ & Maximum effectiveness of flea transmission & 0,3 \\
\hline$\delta$ & Minimum effectiveness of flea transmission (proportion of max.) & 0.25 \\
\hline$\mu_{I}$ & Mortality rate of infected rabbits & $365 / 12$ \\
\hline
\end{tabular}

Table continued on next page... 
Fleas and cats cont.

\begin{tabular}{|c|ll|}
\hline$\mu_{C}$ & Mortality rate of cats & $1 / 4$ \\
\hline$p_{S}$ & Predation rate on susceptible rabbits & 0.0005 \\
\hline$p_{I}$ & Predation rate on infected rabbits & 0.0005 \\
\hline$e_{R}$ & Rabbit conversion efficiency (cats born per rabbit consumed) & 0.015 \\
\hline$r$ & Long-term proportion of rabbits scscueptible to disease & 0.2 \\
\hline$x$ & Time for rabbit susceptibility to disease to decay by half & 20 \\
\hline
\end{tabular}

\section{Birds and Rabbits}

\begin{tabular}{|c|ll|}
\hline Symbol & Definition & Parameter value \\
\hline$\Lambda$ & Constant birth rate of seabirds & 100,000 \\
\hline$\Omega$ & Maximum decrease in seabird births by rabbits (by division) & 10 \\
\hline$\mu_{B}$ & Mortality rate of seabirds & $1 / 15$ \\
\hline$\alpha$ & Maximum per capita growth rate per susceptible rabbit & 2.25 \\
\hline$K$ & Carrying capacity of rabbit population & $0-200,000$ \\
\hline$\mu_{S}$ & Mortality rate of susceptible rabbits & $1 / 1.5$ \\
\hline$k_{B}$ & Constant for logarithmic effect of rabbits on seabird births & 100,000 \\
\hline
\end{tabular}

\section{Simulations}

$\underline{\text { Disease versus predators }}$

\begin{tabular}{|c|ll|}
\hline Symbol & Definition & Value \\
\hline$B_{0}$ & Initial number of seabirds & $1,000,000$ \\
\hline$S_{0}$ & Initial number of susceptible rabbits & 130,000 \\
\hline$I_{0}$ & Initial number of infected rabbits & 0,100 \\
\hline$C_{0}$ & Initial number of cats & 0,350 \\
\hline$\Lambda$ & Constant birth rate of seabirds & 100,000 \\
\hline$\alpha$ & Maximum per capita growth rate per susceptible rabbit & 2.25 \\
\hline$K$ & Carrying capacity of rabbit population & 200,000 \\
\hline$\beta$ & Contact rate of an infected rabbit & $0,0.001,0.003$ \\
\hline$\sigma$ & Maximum effectiveness of flea transmission & $0,3,3$ \\
\hline$\delta$ & Minimum effectiveness of flea transmission (proportion of max.) & 0.25 \\
\hline$\Omega$ & Maximum decrease in seabird births by rabbits (by division) & 10 \\
\hline$\mu_{B}$ & Mortality rate of seabirds & $1 / 15$ \\
\hline$\mu_{I}$ & Mortality rate of infected rabbits & $365 / 12$ \\
\hline$\mu_{C}$ & Mortality rate of cats & $1 / 4$ \\
\hline$p_{B}$ & Predation rate on seabirds & 0.075 \\
\hline$p_{S}$ & Predation rate on susceptible rabbits & 0.0005 \\
\hline$p_{I}$ & Predation rate on infected rabbits & 0.0005 \\
\hline$e_{B}$ & Seabird conversion efficiency (cats born per seabird consumed) & 0.0075 \\
\hline$e_{R}$ & Rabbit conversion efficiency (cats born per rabbit consumed) & 0.015 \\
\hline$r$ & Long-term proportion of rabbits scscueptible to disease & 0.2 \\
\hline$x$ & Time for rabbit susceptibility to disease to decay by half & 20 \\
\hline$k_{B}$ & Constant for logarithmic effect of rabbits on seabird births & 100,000 \\
\hline
\end{tabular}


$\underline{\text { Full model and carrying capacity }}$

\begin{tabular}{|c|ll|}
\hline Symbol & Definition & Value \\
\hline$B_{0}$ & Initial number of seabirds & $1,000,000$ \\
\hline$S_{0}$ & Initial number of susceptible rabbits & 130,000 \\
\hline$I_{0}$ & Initial number of infected rabbits & 0,100 \\
\hline$C_{0}$ & Initial number of cats & 350 \\
\hline$\Lambda$ & Constant birth rate of seabirds & 100,000 \\
\hline$\alpha$ & Maximum per capita growth rate per susceptible rabbit & 2.25 \\
\hline$K$ & Carrying capacity of rabbit population & 200,$000 ; 75,000 ; 12,940$ \\
\hline$\beta$ & Contact rate of an infected rabbit & 0.001 \\
\hline$\sigma$ & Maximum effectiveness of flea transmission & 3 \\
\hline$\delta$ & Minimum effectiveness of flea transmission (proportion of max.) & 0.25 \\
\hline$\Omega$ & Maximum decrease in seabird births by rabbits (by division) & 10 \\
\hline$\mu_{B}$ & Mortality rate of seabirds & $1 / 15$ \\
\hline$\mu_{I}$ & Mortality rate of infected rabbits & $365 / 12$ \\
\hline$\mu_{C}$ & Mortality rate of cats & $1 / 4$ \\
\hline$p_{B}$ & Predation rate on seabirds & 0.075 \\
\hline$p_{S}$ & Predation rate on susceptible rabbits & 0.0005 \\
\hline$p_{I}$ & Predation rate on infected rabbits & 0.0005 \\
\hline$e_{B}$ & Seabird conversion efficiency (cats born per seabird consumed) & 0.015 \\
\hline$e_{R}$ & Rabbit conversion efficiency (cats born per rabbit consumed) & 0.0075 \\
\hline$r$ & Long-term proportion of rabbits scscueptible to disease & 0.2 \\
\hline$x$ & Time for rabbit susceptibility to disease to decay by half & 20 \\
\hline$k_{B}$ & Constant for logarithmic effect of rabbits on seabird births & 100,000 \\
\hline
\end{tabular}

Acknowledgements. The author is grateful to Robert Smith? for his support and guidance on this project. The author is also grateful to Robert Smith? for providing the code for LHS and PRCC. All analysis and figures were done using MATLAB ${ }^{\circledR}$ Release R2015b [15]. 


\section{REFERENCES}

[1] D. M. Bergstrom, A. Lucieer, K. Kiefer, J. Wasley, L. Belbin, T. K. Pedersen, and S. L. CHOwn, Indirect effects of invasive species removal devastate World Heritage Island, J. Appl. Ecol., 46 (2009), pp. 73-81.

[2] D. M. Bergstrom, A. Lucieer, K. Kiefer, J. Wasley, L. Belbin, T. K. Pedersen, and S. L. Chown, Management implications of the Macquarie Island trophic cascade revisited: a reply to Dowding et al. (2009), J. Appl. Ecol., 46 (2009), pp. 1133-1136.

[3] Canadian Museum of Nature, King Penguin, 2016, http://www.nature.ca/notebooks/ english/kingpeng.htm (accessed 2016-08-14).

[4] G. Copson and J. Whinam, Review of ecological restoration programme on subantarctic Macquarie Island: Pest management progress and future directions, Ecol. Manag. Restor., 2 (2001), pp. 129-138.

[5] Department of the Environment, Southern giant petrel, 2005, http://heardisland.antarctica. gov.au/nature/animals-of-himi/flying-birds/southern-giant-petrels (accessed 2016-08-14).

[6] Department of the Environment, Macquarie Island: from rabbits and rodents to recovery and renewal, tech. report, 2014.

[7] O. Diekmann, J. Heesterbeek, and J. Metz, On the definition and the computation of the basic reproduction ratio $R_{0}$ in models for infectious diseases in heterogeneous populations, J. Math. Biol., 28 (1990), pp. 365-382.

[8] J. E. Dowding, E. C. Murphy, K. Springer, A. J. Peacock, and C. J. Krebs, Cats, rabbits, Myxoma virus, and vegetation on Macquarie Island: a comment on Bergstrom et al. (2009), J. Appl. Ecol., 46 (2009), pp. 1129-1132.

[9] G. R. Fulford, X. J. Lee, D. Berman, and G. Hamilton, Interaction Of Myxomatosis And Rabbit Haemorrhagic Disease In Wild Rabbit, 19th Int. Congr. Model. Simul., (2011).

[10] Iowa State University, Rabbit Hemorrhagic Disease, tech. report, 2007.

[11] D. A. Jessup, The welfare of feral cats and wildlife, J. Am. Vet. Med. Assoc., 225 (2004), pp. 1377-83.

[12] P. J. KerR, Myxomatosis in Australia and Europe: A model for emerging infectious diseases, Antiviral Res., 93 (2012), pp. 387-415.

[13] J. Li, D. Blakeley, and R. J. Smith?, The Failure of $R_{0}$, Comput. Math. Methods Med., 2011 (2011), pp. 1-17.

[14] A. J. LotKA, Elements of Physical Biology, Williams and Wilkins, Baltimore, 1925.

[15] MATlaB Release 2015B, The MathWorks Inc., Natick, Massachusetts.

[16] Parks and Wildlife Service, Plan for the Eradication of Rabbits and Rodents on Subantarctic Macquarie Island, tech. report, 2007.

[17] Parks and Wildlife Service, Evaluation Report: Macquarie Island Pest Eradication Project, August 2014, tech. report, 2014.

[18] Parks and Wildlife Service, Animals and Plants, 2015, http://www.parks.tas.gov.au/index. aspx?base $=623$ (accessed 2016-08-14).

[19] Parks and Wildlife Service, Macquarie Island Pest Eradication Project, 2015, http://www. parks.tas.gov.au/?base $=13013$ (accessed 2016-08-14).

[20] Pittsburgh Zoo \& PPG Aquarium, King Penguin, 2015, http://www.pittsburghzoo.org/ ppganimal.aspx?id=63 (accessed 2016-08-14).

[21] B. Raymond, J. McInnes, J. M. Dambacher, S. Way, and D. M. Bergstrom, Qualitative modelling of invasive species eradication on subantarctic Macquarie Island, J. Appl. Ecol., 48 (2011), pp. 181-191.

[22] M. J. Rayner, M. E. Hauber, M. J. Imber, R. K. Stamp, and M. N. Clout, Spatial heterogeneity of mesopredator release within an oceanic island system, Proc. Natl. Acad. Sci., 104 (2007), pp. 20862-20865.

[23] G. W. Roemer and C. J. Donlan, Biology, Policy and Law in Endangered Species Conservation: II. A Case History in Adaptive Management of the Island Fox on Santa Catalina Island, California, Endangered Species UPDATE, 22 (2005), pp. 144-156.

[24] W. E. Sobey, K. M. Adams, G. C. Johnston, L. R. Gould, K. N. G. Simpson, and K. Keith, Macquarie Island: the introduction of the European rabbit flea Spilopsyllus cuniculi (Dale) as a possible vector for myxomatosis, J. Hyg. (Lond)., 71 (1973), p. 299.

[25] A. Terauds, J. Doube, J. McKinlay, and K. Springer, Using long-term population trends of an invasive herbivore to quantify the impact of management actions in the sub-Antarctic, Polar Biol., 37 (2014), pp. 833-843.

[26] N. J. WebB, Growth and mortality in juvenile European wild rabbits (Oryctolagus cuniclus), J. Zool., 230 (1993), pp. 665-677. 